\title{
Auxology: An Update
}

\author{
Michael Hermanussen
}

Altenhof, Germany

- Plotting individual measurements into percentiles or standard deviation score (SDS) scales is superior to immediately plotting the original measurements in centimeters.

- A difference in an individual's maturational tempo from average (percentile crossings) can lead to characteristic height SDS troughs or peaks.

- In puberty, almost half of all height variation is caused by variation in tempo.

\section{Key Words}

Amplitude, growth $\cdot$ Daily height measurements •

Developmental tempo $\cdot$ Height variation $\cdot$ Percentile

crossing $\cdot$ Standard deviation score

\begin{abstract}
Human growth is a dynamic process that needs careful documentation. It is recommended to refer individual growth to a reference population, and to transform and plot individual measurements into percentiles or standard deviation score (SDS) scales. A certain amount of percentile or SDS crossing is a physiological phenomenon that occurs at all ages and relates to the individual changes in developmental tempo. Between early childhood and adulthood, two thirds of all children and adolescents cross $>1$ SD in height. Particularly during pubertal age, SDS crossings can lead to several characteristic patterns such as sharp downward or upward SDS peaks. Upward SDS peaks indicate faster than average tempo, downward SDS peaks indicate slower than average tempo. Almost half of the pubertal height variation consists of variation in tempo. Applying functional data analysis and principal component analysis, the two main sources of height variance, i.e. tempo and amplitude, can be statistically separated
\end{abstract}

and quantified. This review aims to renew some of the old debates on how to assess growth, and to facilitate forthcoming consensus upon how to appropriately evaluate height and changes in height at brief and clinically relevant intervals.

\section{Documenting Growth}

Growth references describe how children grow.

Growth standards describe how children should grow.

Cross-sectional distance charts provide percentiles for height, weight and other anthropometric parameters against age.

Velocity charts provide percentiles for the differences in height, weight and other parameters against a standard time interval (usually 12 months).

Tempo-conditional charts provide percentiles that allow for distinguishing between early- or late-maturing children.

Human growth is a dynamic process that needs careful documentation. It is usually visualized by plotting individual measurements on so-called growth or distance charts. These charts are derived from national cross-sectional surveys, or they can synthetically be constructed

Prof. Dr. Michael Hermanussen

Aschauhof 3

DE-24340 Altenhof (Germany)

Tel. +49 4346601 137 or +49 435141738 , Fax +49 4346368484

E-Mail hermanussen.aschauhof@t-online.de 
from representative local cohorts at selected ages, which in terms of cost-benefit appears to exhibit significant advantages [1]. Growth charts are used to compare an individual's height, weight and other parameters to an applicable reference population. In fact, evaluation of individual growth, particularly using SD scores (see below), heavily depends on the appropriateness of the reference population data. Emphasizing the similarity in early childhood growth among diverse ethnic groups, some authors distinguish between growth references that describe how children grow and growth standards that rather describe how children should grow [2]. Standards for child growth are now offered around the world (http:// www.who.int/childgrowth/1_what.pdf).

Differences between two measurements, divided by a time interval between them (preferably 12 months; 6-month intervals can be reliable if seasonal variation in growth rates are small), can be plotted on so-called velocity charts. Velocity charts are usually constructed from longitudinal surveys with multiple annual or semi-annual measurements. Velocity charts are usually tempo unconditional, i.e. they consist of height/weight differences, for example, obtained from children grouped according to calendar age, regardless of their developmental tempo, i.e. regardless of their maturational state (biological age). Tempo-conditional charts allow for considering differences in the individual developmental tempo, but they can only be applied when additional information on developmental tempo is available, i.e. when clinical signs of puberty are visible, or when bone age, or the age of peak height velocity of that subject, are known. As this information is not usually available, tempo-conditional growth charts are rarely used in clinical routine.

\section{Measurement Accuracy}

The technical error indicates the deviation of an individual observation from its true value, caused by the unreliability of the instrument and the investigator's skill. The technical error is expressed as the standard deviation of repeated measurements obtained at the same occasion. Under optimal conditions, the technical error of height amounts to some $3 \mathrm{~mm}$.

Knemometry (from Greek $\eta \kappa \nu \eta \mu \eta$, the lower leg) denotes a technical device for very accurate measurements of the distance between knee and heel of a sitting child or adolescent. The measurement error of knemometric measurements is $<160 \mu \mathrm{m}$. Knemometry illustrates growth at intervals of few weeks (short-term growth).

Distance and velocity charts are common tools in pediatric practice, but their validity depends on measure- ment accuracy. The technical error of height measurements varies considerably with the investigator's skills and usually ranges between 3 and $4 \mathrm{~mm}$ in the standing child. In 1929, Boyd [3] found 'that 75 per cent of the average deviations (from a straight line) are less than $0.4 \mathrm{~cm}$ for measures of standing height'. In view of an average prepubertal annual growth rate of almost $6 \mathrm{~cm}$, a technical error of $3 \mathrm{~mm}$ appears small. Yet when considering height velocities, one must remember that a height velocity always consists of two measurements and is afflicted with the measurement error of both measurements.

The reliability of a single measurement can be improved when the measurement is repeated. Under routine conditions, this may not always be necessary, but when height increments need to be documented at short term, e.g. some 2 or 3 months before and during the initiation of a growth therapy, assessing growth may require some additional tools: one may either improve measuring accuracy (improving quality) [4] or one may rigorously increase measurement frequency (improving quantity). Several approaches have been published to overcome this dilemma. Accuracy can be increased by applying highly accurate technical devices such as knemometry [5]. Knemometry measures the lower leg length and significantly shortens the time for estimating growth rates to periods of a few weeks. It is still being used as an integral part of the safety assessments of new inhaled glucocorticoids and inhalation devices in children with asthma [6]. Yet, the technique is sophisticated and expensive.

Measurement accuracy also needs to take the circadian variation into consideration. As most height loss occurs in the morning, afternoon clinic appointments would be preferable [7]. For evaluating short-term growth, Tillman and Clayton [8] recommend that children should be measured by a standard stretch technique between 18:00 and 21:00 $\mathrm{h}$, when the diurnal variation in height is smallest.

The technical error of weight measurements ranges within 100-200 g depending on the measuring device, but throughout the day tends to vary considerably due to eating and defecation. Weight measures are most consistent when obtained before breakfast.

\section{Growth Spurts and Height Velocity}

Mini-growth spurts (also referred to as saltation and stasis) are rapid changes in height velocity within intervals of 2-10 days with average peak height velocity of $85 \mu \mathrm{m} / \mathrm{h}$.

Catch-up growth describes compensatory growth after periods of previous growth deceleration. 
Human growth is characterized by marked changes in height velocity [9]. Rapid intrauterine growth with maximum height velocity in the 3rd trimenon is followed by a postnatal spurt immediately after birth, a mid-growth spurt at the age of 6-7 years, and a pubertal growth spurt with peak height velocity during mid-puberty around the age of 13-14 years in boys, and 11-12 years in girls. In addition, minor spurts exist. Cyclic variation in height velocity at approximately 2 -year periodicity has been described by Butler et al. [10], and short-term changes in height velocity occur at even smaller intervals of few days. The characteristic pattern of chaotic series of rapid shortterm growth changes, called mini-growth spurts, with average peak height velocity of $85 \mu \mathrm{m} / \mathrm{h}$ at intervals of $2-10$ days, followed by periods of growth arrest or diminished growth velocity has extensively been studied in the lower leg (knemometry) of rodents [11] and in newborn and premature infants [12]. As this pattern consists of jumps followed by stagnation, some authors refer to it as saltation and stasis [13]. During periods of illness, starvation [14, 15] or social deprivation, height velocity tends to decelerate, but usually rises again and compensates for the previous losses (catch-up growth [16]) when the unfavorable situation has been overcome.

\section{Some Practical Issues in View of Measurement Accuracy and Short-Term Changes in Growth}

High-frequency measurements are measurements performed by conventional devices, at daily or near-daily intervals. They have almost exclusively been used for scientific purposes.

Daily 'home-made' measurements can be performed by the parents, but parents need careful instructions and the measurements require some additional statistical treatment before clinical consequences can be drawn.

Clinical routine usually warrants fast decisions. However, how to provide reliable measures of height velocity when both measurement error and rapid changes in short-term growth complicate the measurements? We have mentioned improving quality versus improving quantity. Accurate lower leg length measurements using knemometry is elaborate. Increasing measurement frequency using conventional body height measurements including an appropriate statistical treatment is simple. High-frequency measurements, though to date almost exclusively being used for scientific purposes [17], have been found practicable and similarly predictable in documenting treatment success in growth hormone-deficient children [18]. In recent years, several publications have indi-
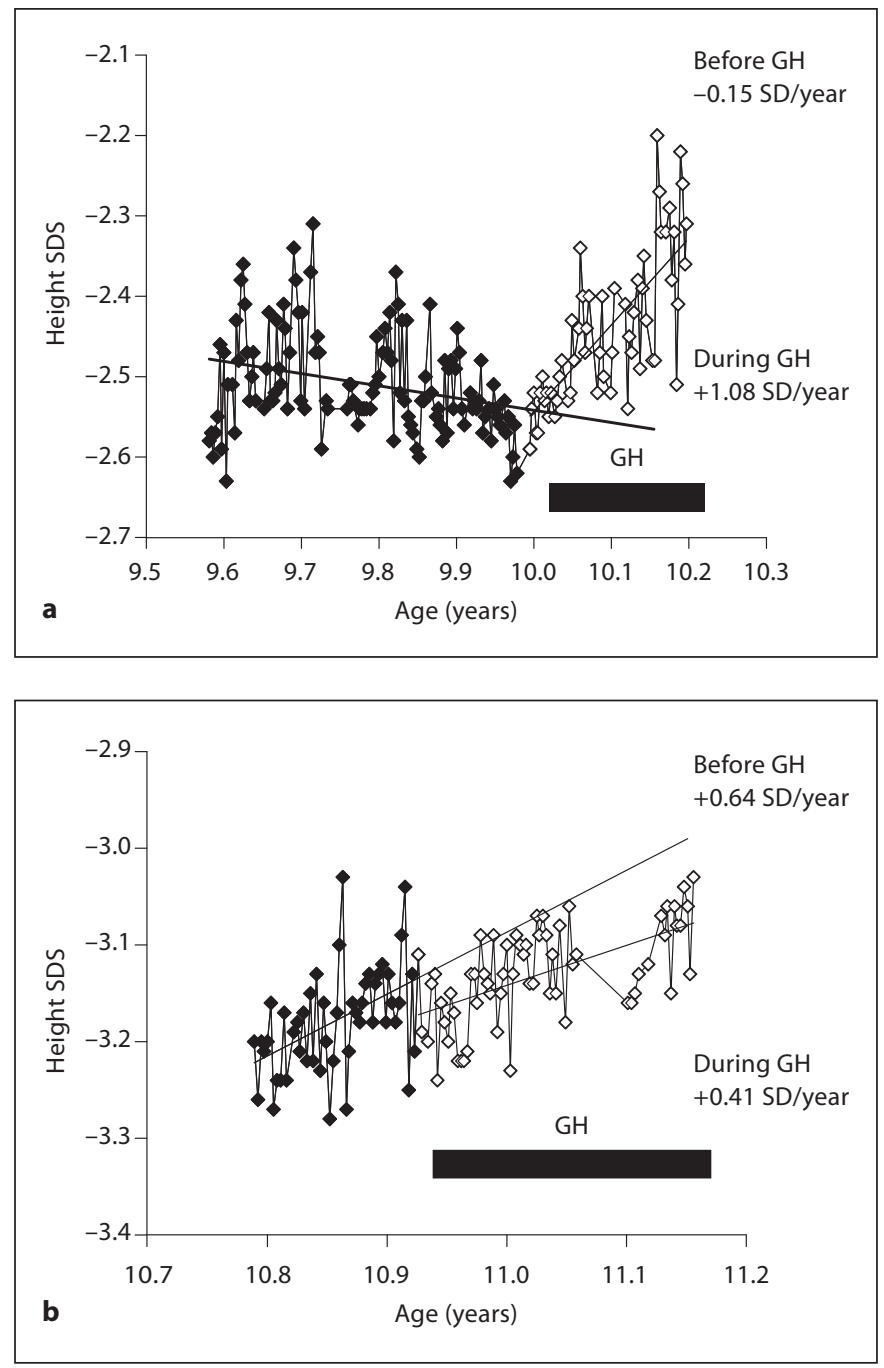

Fig. 1. Growth dynamics of 2 children with idiopathic growth hormone $(\mathrm{GH})$ deficiency before $(\checkmark)$ and during the treatment with $\mathrm{GH}(\diamond)$ for 3 months. One child clearly responds to $\mathrm{GH}$ and significantly increases in height SD from $-0.15 \mathrm{SD} /$ year before to $+1.08 \mathrm{SD}$ /year during the treatment $(\mathbf{a} ; \mathrm{p}<0.05)$, providing supporting evidence for successful GH treatment within 3 months. The other child fails to respond to GH (b). Height SDS rises with $0.64 \mathrm{SD} /$ year already before starting the treatment and rather appears to decrease during $\mathrm{GH}$ administration with $0.41 \mathrm{SD} /$ year.

cated that clinical information may even be drawn from daily parental 'home-made' measurements. Although such measurements are less accurate than measurements done by trained personnel, the plain quantity of information offers an interesting alternative for both practical and accurate growth monitoring $[18,19]$ - provided that parents have carefully been instructed before and the 
measurements undergo additional statistical treatment before clinical consequences are drawn. Figure 1 illustrates growth dynamics of 2 children treated with growth hormone. One child significantly improves and gains height SDS (fig. 1a), whereas the other fails to clearly respond to the treatment (fig. 1b).

Caino et al. [17] developed an appealing approach (jump-preserving smoothing technique) for the analysis of serial height data obtained at daily intervals. The technique was derived from so-called 'edge-related smoothers' that have been developed for image processing [20]. The technique reduces the variability in stature that is caused by measurement error and helps revealing the true underlying growth process.

\section{What Is Normal?}

Little disagreement on what is normal, and what is not normal, exists when considering plain stature or weight. References or standards for body height, weight and other parameters provide quite indisputable statements about whether a child is too tall, too short, too thin or too heavy. Similarly little disagreement exists when considering height velocity obtained at standard intervals. Yet the majority of children presented for growth assessment has either not at all been measured before the clinical statement is due or not at standard intervals. However, the question remains: what is normal in respect to growth velocity and short-term growth, and ever since it has been a matter of debate.

In spite of the characteristic patterns of short-term growth [4], normal values for short-term growth have never been published. Since sophisticated technical devices or appealing statistical approaches that lack normal values are not useful for general clinical practice, most of us still hang around with some decade-old traditional annual height velocity chart. This is unsatisfying, and we urgently long for alternatives. This review aims to renew some of the old debates on how to document growth, and to facilitate forthcoming consensus upon how to appropriately evaluate height and changes in height at brief and clinically relevant intervals.

The first step in considering whether a given growth pattern is normal or abnormal is to abandon some of the traditional ideas about growth chart and growth rates. While plotting height on conventional growth charts and relying on strictly annual or semi-annual measurement intervals is cumbersome, the new concepts developed in the recent years make it much easier to assess growth and require markedly less rigid schedules than hitherto practiced.

The first step is transforming scales. We transform the original measurement scale, expressed in centimeters, kilograms or kilograms per meter squared, and refer the individual measurements to a reference population plotting them into percentiles or SDS scales.

\section{Standard Deviation Scores}

Canalized or parallel to percentile growth indicates a stable position in height ranks among peers of the same age. Parallel to percentile growth is characterized by horizontal SDS patterns.

The LMS method adjusts for skewness and allows parameters that are not normally distributed, such as weight or body mass index, to be expressed as an exact percentile or an SD score. $M$ stands for mean, $S$ stands for standard deviation, and $L$ stands for the Box-Cox power that is required to transform the skewed data to normality.

The mean change in height SDS is zero.

The standard deviation in height SDS change depends on age, and is given by $\sqrt{2}(1-r)$, where $r$ is the correlation between SDS $_{1}$ and $\mathrm{SDS}_{2}$.

Height, body weight, body mass index and many other anthropometric parameters can either be described in absolute terms (the measurement scale, e.g. $\mathrm{cm}$, kg or $\mathrm{kg} / \mathrm{m}^{2}$ ) or they can be referred to a reference population using percentiles or, since they correspond one to one, using an SDS scale. Transforming and plotting individual measurements into percentiles or SDS scales has been found favorable for clinical purposes. Transforming height measurements into height SDS is trivial as the biological parameter height is normally distributed. Weight and body mass index distributions, however, are skewed and need adjustment. The LMS method is strongly recommended for this purpose [21,22]. Most modern growth references and growth standards use LMS technology (e.g. WHO [23] and UK reference curves [24]). The LMS method is superior to simply counting percentiles. Counting percentiles was done in historic growth studies, but is considered uncomfortable as it often results in irregularly warped charts. For clinical purposes we need appropriate references, appropriate in the sense that they provide smoothed means, smoothed standard deviations and smoothed Box-Cox power or skewness for all ages between birth and maturity.

Why SDS scales? Growth charts conventionally record cross-sectional (distance) information, but they can be extended to monitor growth rate over time (velocity) [25]. When plotting SDS against age, serial measurements of an 
individual's height, weight or any other anthropometric parameter usually appear as fairly horizontal lines. Horizontal lines can easier been analyzed, and have long been recommended as a better way to present growth data [26]. Using SDS plots, growth velocity manifests as the rate of change in SDS rather than the rate of change in the measurements themselves. Serial SDS illustrate growth dynamics. Figure 2 exemplifies serial SDS of 15 male and 15 female individuals of the FirstZürich Longitudinal Growth Study [27]. The upper three panels of figure $2 \mathrm{a}$ and $\mathrm{b}$ (males and females, respectively) present fairly horizontal patterns indicating canalized or parallel to percentile growth. The other individuals cross percentiles, some show downward percentile crossings and downward peaks, other show upward percentile crossings and upward peaks. Downward percentile crossings indicate slower than average, upward percentile crossings faster than average height velocity. The characteristic peaks usually occur during puberty.

In 1998, Cole [25] summarized statistical considerations and practical issues of changes in height SDS. He showed that serial SDS are far more attractive than height or weight measurements plotted on velocity charts. Changes in SDS over a time interval can be used to calculate growth velocity and in the same instant to estimate whether changes are acceptable. Mean and variance (var) of changes in SDS are given by

$$
\begin{aligned}
& \text { mean }\left(\mathrm{SDS}_{1}-\mathrm{SDS}_{2}\right)=\text { mean }\left(\mathrm{SDS}_{1}\right)-\text { mean }\left(\mathrm{SDS}_{2}\right)=0 \\
& \operatorname{var}\left(\mathrm{SDS}_{1}-\mathrm{SDS}_{2}\right)=\operatorname{var}\left(\mathrm{SDS}_{1}\right)+\operatorname{var}\left(\mathrm{SDS}_{2}\right) \\
& -2 \operatorname{cov}\left(\mathrm{SDS}_{1}, \mathrm{SDS}_{2}\right)=2(1-r),
\end{aligned}
$$

where $r$ is the correlation between $\mathrm{SDS}_{1}$ and $\mathrm{SDS}_{2}$, and can be used to establish normal values for velocity SDS. In 1997, Cole [22] published normal values for velocity SDS for the age range of 2-9 years based on the French longitudinal growth study. Normal values for velocity $S D S$ for all ages are still to be published.

\section{Why Do Percentiles Cross?}

Upward SDS peaks shortly after age at peak height velocity $(A P H V)$ are characteristic for early-maturing adolescents.

Downward SDS peaks shortly before APHV are characteristic for late-maturing adolescents.

Age at peak height velocity (APHV) can be used as an estimate for maturational tempo.

How much percentile or SDS crossing is acceptable? Does it depend on age, or on the starting percentile? Percentile or SDS crossing is a ubiquitous and certainly phys- iological phenomenon in early human growth, but actually occurs at all ages. Between the age of 2 years and adulthood, two thirds of all subjects of the First Zürich Longitudinal Growth Study crossed $>1$ SD [28]. Most crossings are not just simple rises or drops in SDS. Particularly during pubertal age, characteristic patterns occur consisting of sharp downward or upward SDS peaks. As shown in figure 2, upward SDS peaks indicate faster, downward SDS peaks indicate slower than average tempo.

Yet determining maturational tempo from longitudinal data, even after transforming serial height into serial height SDS, remains difficult. In view of a number of characteristic inflexion points of the individual growth velocity curve, such as the moment of minimum height velocity shortly before the onset of the adolescent growth spurt, or the moment of maximum height velocity during the adolescent spurt, several mathematical models have been applied since to describe these characteristics, one of the most well-known being Preece-Baines modeling [29]: five parameters describe growth in stature from age 2 to maturity and depict the 'age at take off' - the moment when the adolescent growth spurts starts - and the APHV in mid-puberty. The model thus determines two characteristic states of an individual's maturation. We also used this technique to determine $A P H V$ and thereby to estimate maturational tempo of the individuals shown in figure 2. Figure 2 illustrates that in early-maturing adolescent boys [ $\mathrm{n}=285$ (APHV 12.4 years), 115 (APHV 12.5 years) and 341 (APHV 11.9 years)] with early APHV, upward SDS peaks tend to occur shortly after $A P H V$, whereas in late-maturing adolescent boys [ $\mathrm{n}=250$ (APHV 14.8 years), 328 (APHV 15.7 years) and 197 (APHV 15.8 years)] downward SDS peaks tend to occur shortly before APHV. Figure 2 illustrates that serial height SDS can indicate characteristic states of an individual's maturation. The concept considerably simplifies depicting growth velocity, and it immediately visualizes to what extent the description of height intermingles with developmental or maturational tempo.

(For figure see next pages.)

Fig. 2. Serial height SDS (left scale) and height percentiles (right scale) of a sample of 15 male (a) and 15 female individuals (b) of the First Zürich Longitudinal Growth Study [27]. Subject numbering refers to the original numbering of the study cohort. In order to relate maturational tempo and SDS peaks, height of the individuals were modeled by Preece-Baines [29] analysis. APHV are indicated by vertical bars and given in decimal years ([28], reprint by courtesy of the publisher). 


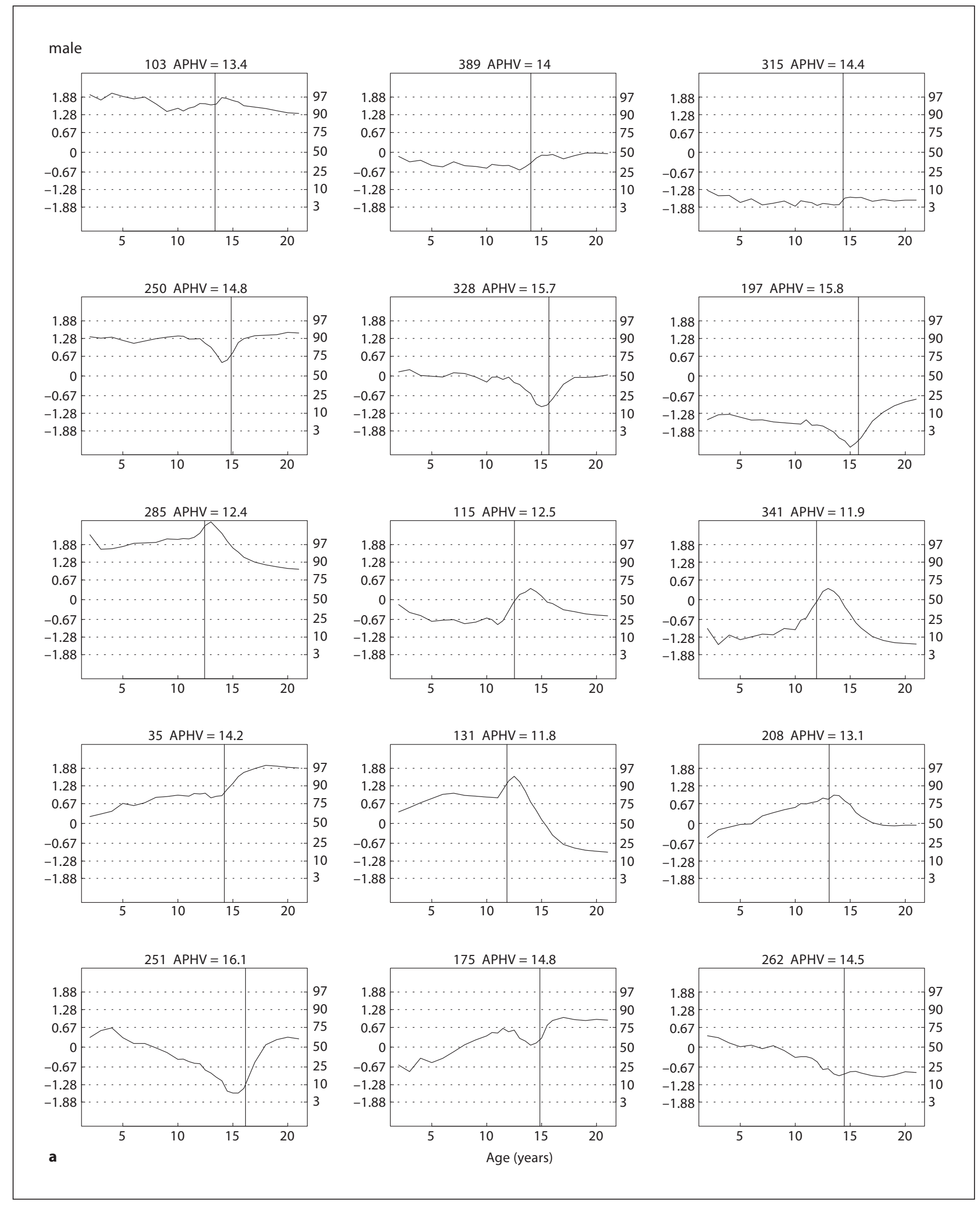



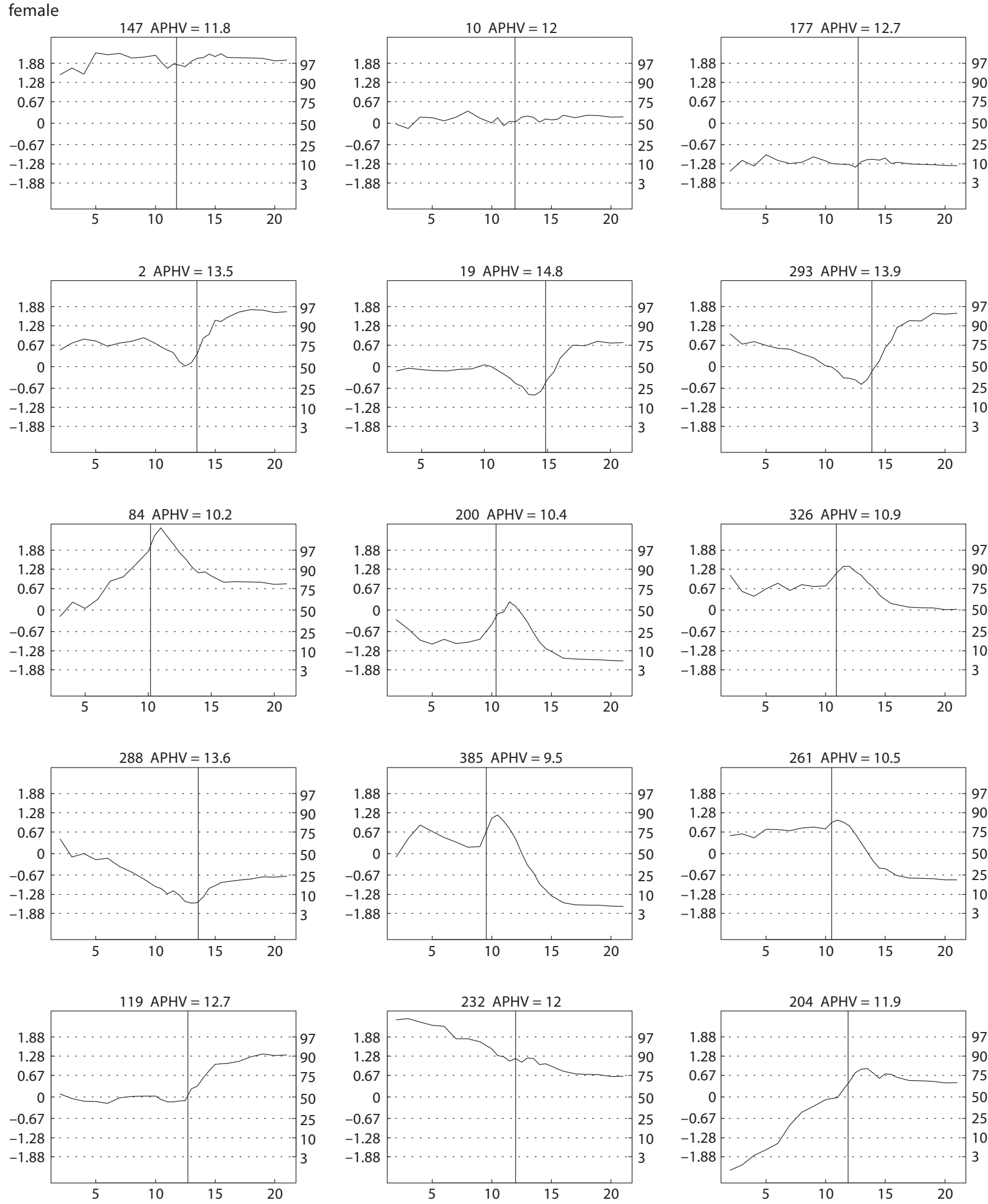

b
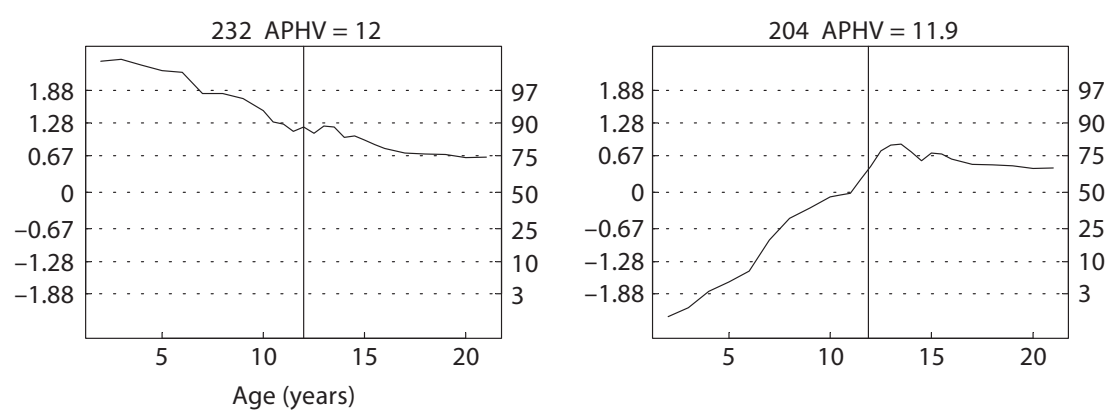
In fact, height and height increment greatly depend on tempo [30] as tempo variation is an important feature of individual growth patterns. The term growth velocity is a semantic chimera. It does not simply reflect 'increment in stature' when reference is made to standard time-related patterns of growth; the term is a conglomerate of both the increment in stature and the progress in maturation. This leads us to an important new question: Why not explicitly study tempo, and why not separately assess the increment in amplitude and the progress in maturation, i.e. the tempo at which an individual matures?

\section{Amplitude Variation versus Tempo Variation}

Being tall or short both depends on increments in amplitude (members of tall grown families tend to be tall at all ages) and on developmental tempo (early-maturing children tend to appear taller than later-maturing peers of the same calendar age).

The rigid metric of physical time is not directly relevant to the internal dynamics of growth.

In puberty almost half of all height variation is caused by tempo variation.

Good clinical practice already attempts to separate the two sources of variation: the tempo and the amplitude variation. X-rays of the left hand are commonly used to assess skeletal maturity as an all-age indicator of tempo. During adolescence, the clinical stages of puberty may additionally be used to indicate the state of maturity. Yet, in contrast to the metric scale for height $(\mathrm{cm})$ and the metric scale for physical time (age in years), there is no apparent metric scale for maturation. In the early 1960s, studying hip joints, Hewitt and Acheson [31, 32] used a scoring system and found a more rapid increase in unweighted bone scores at puberty than before, and showed that the same would apply to the hand and wrist. Tanner et al. [33] converted skeletal maturity into a maturity score, and showed that mean maturity score increments per chronological year differed throughout childhood and adolescence, with sharp increments/year in RUS (radius, ulna and short bones) scores during mid- and endpubertal age. Maturity scores exhibit significant gender dimorphism, with girls scoring approximately 2 years earlier than boys. Unfortunately, the scores for skeletal maturity were later turned back into male and female 'bone ages', muddling up mean annual developmental progress of the reference population, 'calendar age' and the individual progress in maturation.

This uncomfortable semantic confusion still persists. Although estimating an individual's bone age causes no problems per se, major problems arise when describing bone age progression. Maturity score advancements depend on age, and they differ between individuals. Simple ratios such as $\Delta$ bone age/ $\Delta$ chronological age, which have often been used in pediatric endocrinology to characterize a mismatch between calendar time and bone age progression, ignore that the metric of physical time differs from the internal dynamics of growth causing awkward and age-dependent artifacts. Such ratios should always be questioned.

Significant progress in longitudinal growth analysis was made using the methods of functional data analysis and principal component analysis [34]. Functional data analysis provides a new statistical tool to separate the two sources of variation in height increment as it can differentiate between the variation in altitude (amplitude variation) and the variation in developmental progress (tempo variation). Principal component analysis determines amplitude and tempo variation separately by so-called principal components.

We recently extended this concept by combining principal component analysis and maximum likelihood principle for growth modeling [35]. In girls, pubertal height variation appears to consist of $60 \%$ amplitude variation and $40 \%$ variation in tempo. In boys we find an almost $50 / 50 \%$ ratio of amplitude and tempo [35]. The findings closely match estimates based on skeletal maturation [30]. The greatest amplitude component explaining 91\% of the amplitude variance corresponds to the trivial clinical evidence that children who are tall/short early in life will stay tall/short and end up tall/short. The greatest tempo component explaining two thirds of all tempo variance corresponds to the similarly trivial evidence that children who are fast-/slow-maturing early in life stay fast-/slow-maturing throughout most of their development and reach final height early/late. Although the analysis solely utilizes height data, the statistical estimates of tempo significantly correlate with the occurrence of clinical signs of puberty.

Combining principal component analysis and maximum likelihood principle for growth modeling not only parallels and supports well-known clinical evidence, it also enables for the first time quantifying the proportion of amplitude and tempo in measured body height.

\section{Illness and Growth}

During illnesses and starvation, height velocity usually decelerates, but in many cases it remains controversial whether it is the amplitude or the tempo component 

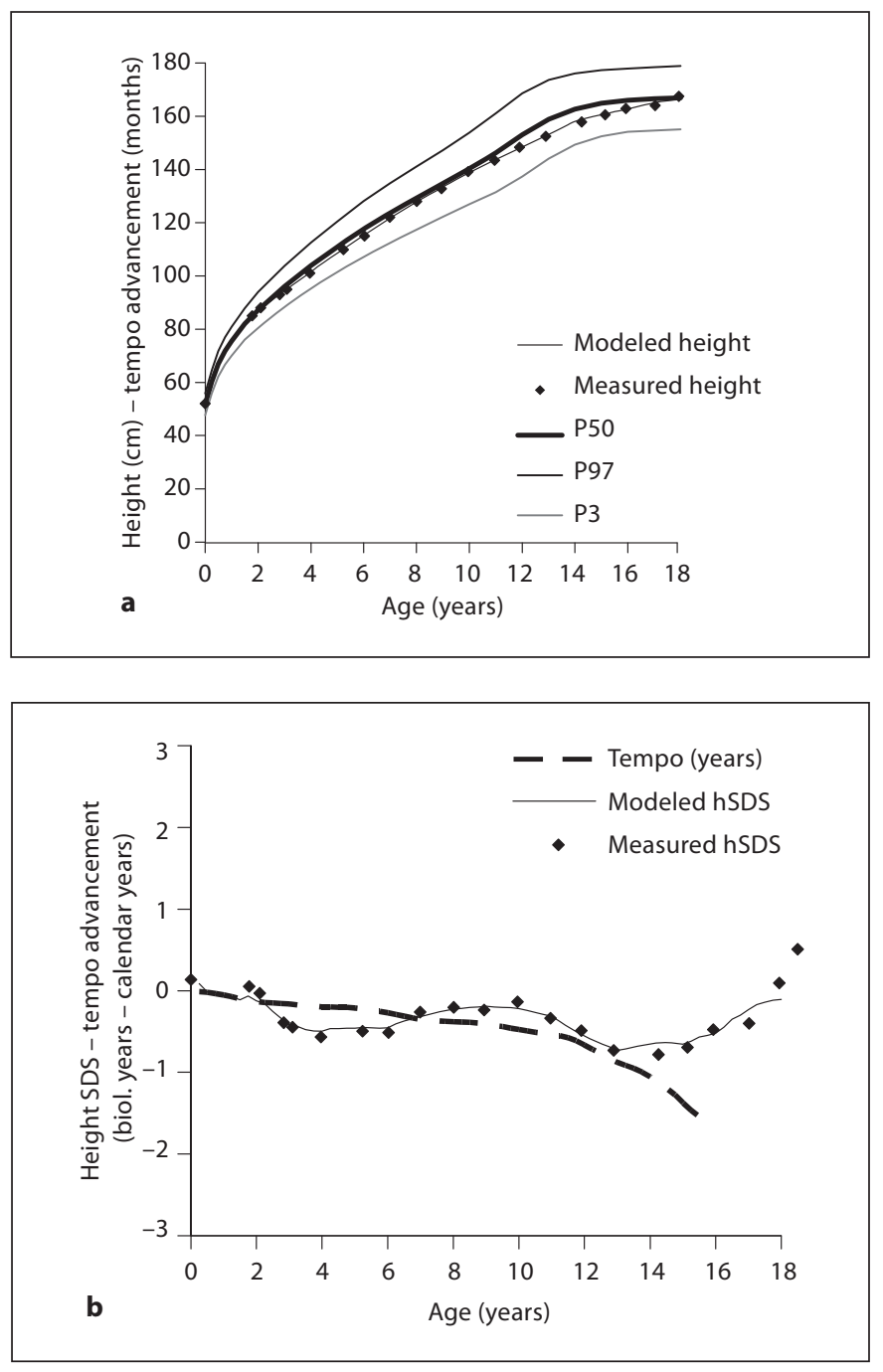

Fig. 3. a Measured height, modeled height [35] and three common percentiles (P50, P3 and P97) in a late-maturing CF female. Downward percentile crossing is obvious. b Tempo advancement (tempo) and height SDS of the same child. The tempo steadily declines below physical time and reaches -1.6 years at the calendar age of 16 . The tempo retardation nicely parallels the decrease in height SDS.

that is disturbed. Whereas height velocity deceleration due to tempo deceleration will lengthen the child's developmental period with no impairment in adult height, height velocity deceleration due to a reduction in amplitude will persist and eventually lead to short adult height.

In contrast to common clinical opinion, many chronic illnesses associated with short stature in childhood do in fact not touch the amplitude component of growth. Aswani et al. [36] showed that even in cystic fibrosis (CF), short stature in childhood results from tempo decelera-

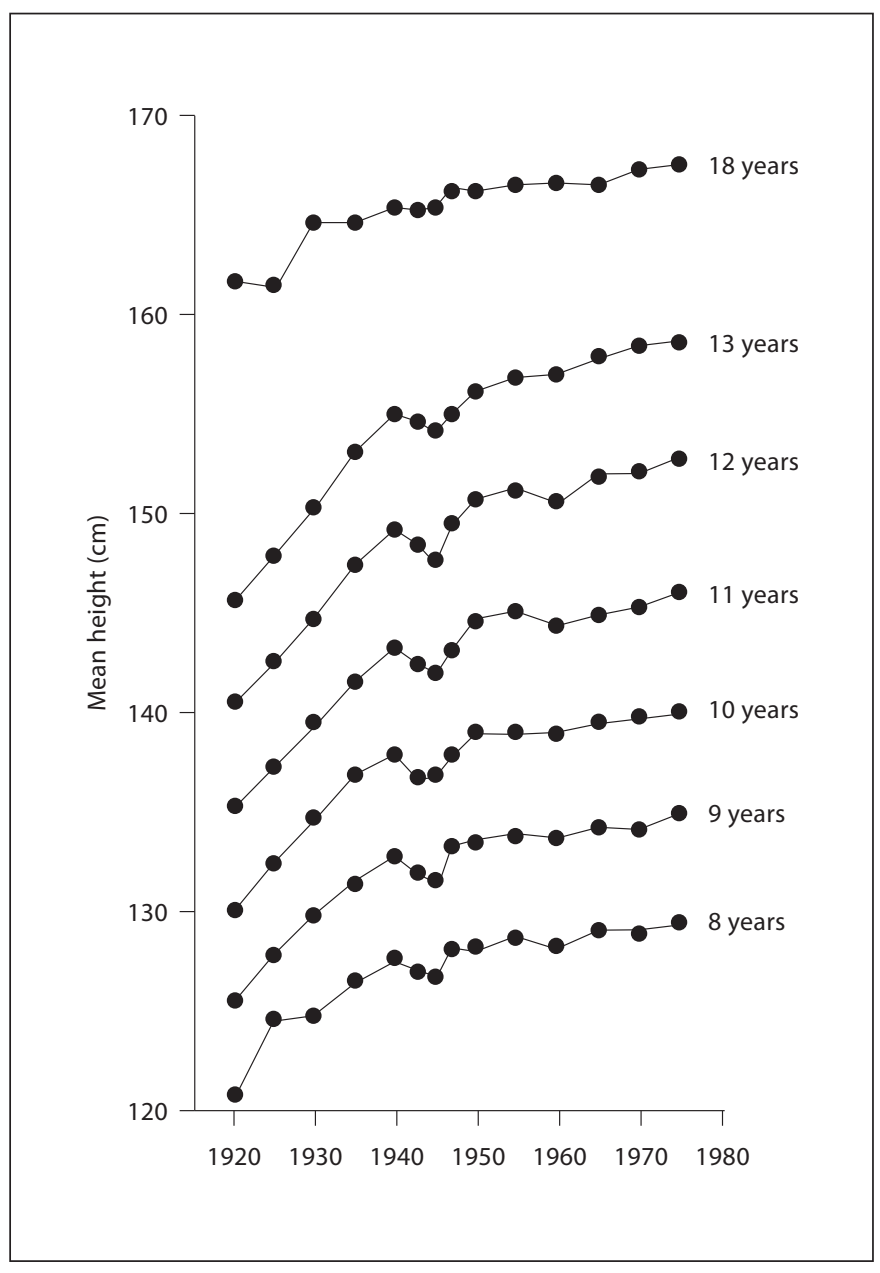

Fig. 4. Mean height of Oslo school girls between 1920 and 1975 ([38], reprint by courtesy of the publisher). Marked reduction in height is obvious in cohorts that suffered from starvation during the German occupation of World War II. After starvation, catchup growth occurred immediately with no evidence of persistent growth impairment. Height in 18-year-old females (near final height) appeared unaffected by the starvation. The apparent height losses in the 8-, 9- and 10-year-old cohorts of 1942 have disappeared in the 18-year-old cohorts of 1950, 1951 and 1952 (same individuals).

tion. Although in CF patients suboptimal peak height velocity and late pubertal growth were influenced by disease severity, they eventually achieved normal final height. Wiedemann et al. [37] stated that in a group of 4,306 CF patients, height $\mathrm{z}$-scores increased with age, and normal means for height were reached in the adult age group. Figure 3 illustrates a typical CF patient with an up to 1.6-year tempo deceleration during puberty, but an appropriate final height. 

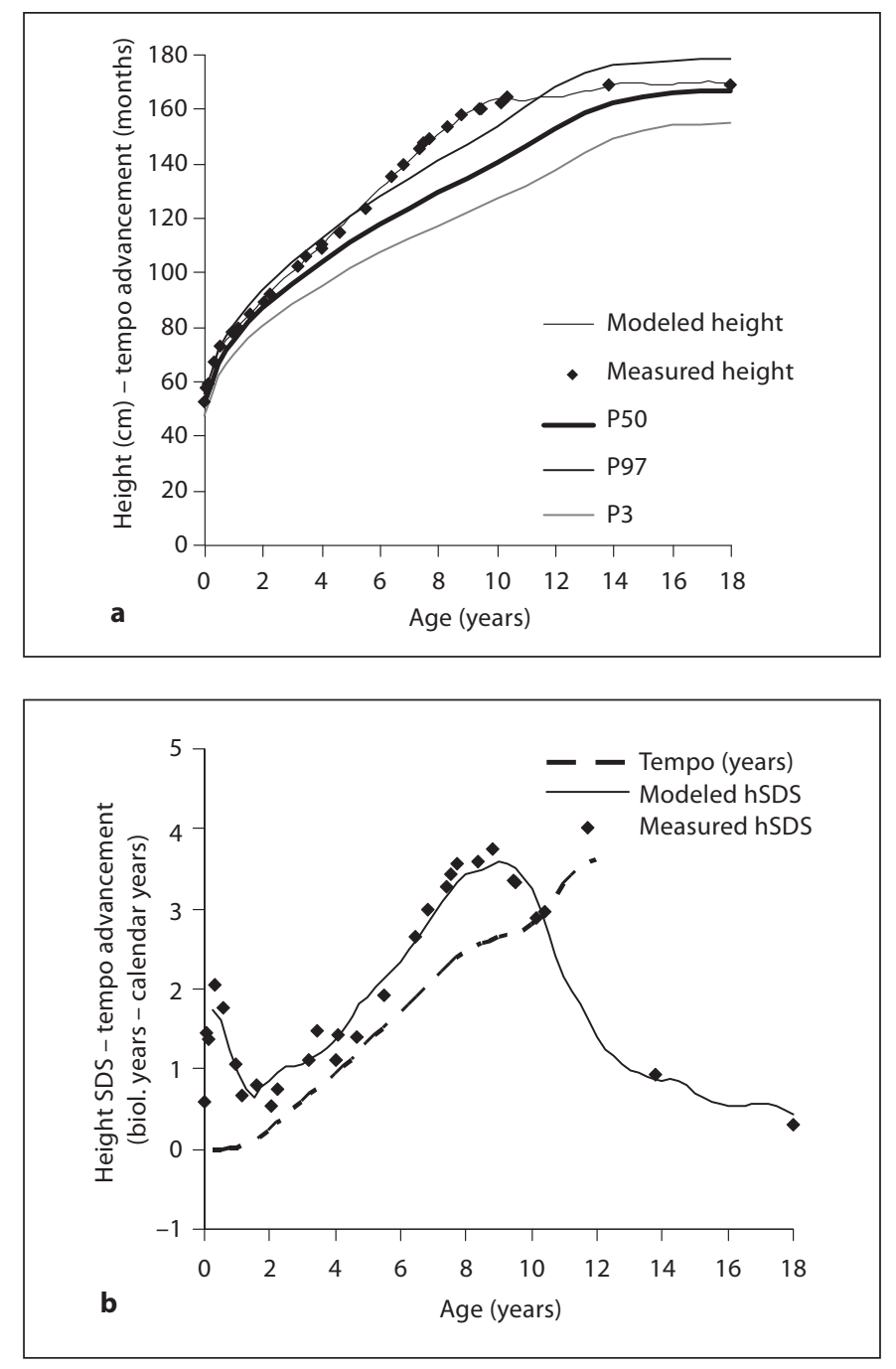

Fig. 5. a Measured height, modeled height [35] and three common percentiles (P50, P3 and P97) in a female patient with congenital adrenal hyperplasia and advanced developmental tempo. Upward percentile crossing during prepubertal age is obvious. b Tempo advancement (tempo) and height SDS (hSDS) of the same child. The tempo steadily advances over physical time and reaches a plus of $>3$ years at the calendar age of 12 . The tempo advancement parallels the increase in hSDS.

Similar observations have been published about starving populations. Rich historic evidence illustrates that caloric restriction primarily affects the tempo, and to a much lesser extent height. During the German occupation of World War II, Oslo schoolgirls had marked growth impairment coinciding with the period of social disaster [38], but rapidly caught up and achieved normal adult stature (fig. 4). Similar phenomena have been observed in German school boys during and after World War II [39].
Patients with congenital adrenal hyperplasia often show accelerated developmental tempo. Figure 5 illustrates a girl with congenital adrenal hyperplasia with a $>3$-year tempo acceleration. Principal component analysis and maximum likelihood principle illustrate how closely both height SDS and tempo correspond to each other.

\section{How to Assess Growth?}

Single height measurements are inadequate to provide clinically meaningful information on stature and growth.

Series of height measurements are indispensable.

\section{Assessment of Stature}

In order to fully understand the dynamic process of height achievement in a certain child, we need separate estimates of amplitude and tempo. As height measurements at strict annual intervals are not required, we can utilize all accessible previous measurements regardless of their intervals. These measurements are all transformed into SDS scales. Thereafter, we analyze SDS patterns.

\section{Assessment of Short-Term Growth}

When evaluating short-term growth dynamics, e.g. immediately before and shortly after initiating growth therapies, we either apply very accurate measuring techniques such as knemometry or we use high-frequency measurements, practically done as daily 'home-made' measurements by the parents, and thereafter apply appropriate statistics on the SDS scale. Simple questions such as: does this child profit from a grow th hormone treatment may then be answered by simply evaluating linear regression lines before and during the treatment, as exemplified in figure 1. More complex questions such as: how do the minimal growth spurts look like may require more sophisticated statistics such as a jump-preserving smoothing technique [17, 40].

\section{Assessment of Developmental Tempo as an Integral}

Part of Assessing Child and Adolescent Growth

Three techniques are currently available for assessing developmental tempo: (1) assessing skeletal maturity and in adolescents the state of pubertal maturity, (2) plotting serial measures of height SDS and searching for characteristic SDS patterns such as percentile crossings and SDS peaks, or (3) applying principal component analysis. 
(1) Assessing skeletal maturity has recently been automatized by Thodberg et al. [41] and Thodberg [42] with surprisingly good results as it appears largely independent of the investigator's skill. It remains to be established whether this methodology may replace traditional technology (e.g. Greulich and Pyle, Bayley and Pinneau, and Tanner and Whitehouse).

Pubertal signs are also valid estimators for maturity, but they can only be used in adolescent individuals.

(2) Plotting series of height SDS allows rough but quick estimates of developmental tempo [28]. Plots of serial height SDS are superior over serial plots of height velocity.

(3) Functional data analysis and principal component analysis can statistically separate and quantify variation in tempo and variation in amplitude. Although an Internet portal is currently available to process small amounts of height data (www.willi-will-wachsen. com) [43], there is certainly an urgent need of practical solutions for worldwide general use of such techniques.

\section{Conclusion}

Growth assessment is not trivial as it needs separating the variation in tempo from the variation in amplitude, but some new concepts have led to a number of simplifications that may markedly facilitate assessing growth, such as eliminating standard intervals for determining growth rates and transforming measurements on SDS scales. A combination of high-frequency measurements and new statistical approaches for analyzing serial height SDS can be applied for efficient assessments of short-term growth dynamics. This review aims to renew some of the old debates on how to assess growth and to facilitate forthcoming consensus upon how to appropriately evaluate height and changes in height at brief and clinically relevant intervals.

\section{Acknowledgments}

This work was supported by the Deutsche Gesellschaft für Auxologie and Norddeutsches Zentrum für Wachstumsforschung. Thanks are due to Dr. Wiedemann, Institut für medizinische Informatik und Biometrie, Dresden, and Dr. Henn, Mucoviscidose Ambulanz, University of Leipzig, for providing growth data of cystic fibrosis patients.

\section{References}

-1 Hermanussen M, Assmann C, Tutkuviene J: Statistical agreement and cost-benefit: comparison of methods for constructing growth reference charts. Ann Hum Biol 2010;37:5769.

2 Garza C, de Onis M: Rationale for developing a new international growth reference. Food Nutr Bull 2004;25(1 suppl):S5-S14.

$\checkmark 3$ Boyd E: The experimental error inherent in measuring the growing human body. Am J Phys Anthropol 1929;13:389-432.

$\checkmark 4$ Hermanussen M: The analysis of short-term growth. Horm Res 1998;49:53-64.

$\checkmark 5$ Hermanussen M: Knemometry, a new tool for the investigation of growth. A review. Eur J Pediatr 1988;147:350-355.

6 Wolthers OD: Methodology and implications of knemometry in growth assessment of inhaled glucocorticoids. Pediatr Allergy Immunol 2009, E-pub ahead of print.

7 Voss LD, Bailey BJ: Diurnal variation in stature: is stretching the answer? Arch Dis Child 1997;77:319-322.

$\checkmark 8$ Tillmann V, Clayton PE: Diurnal variation in height and the reliability of height measurements using stretched and unstretched techniques in the evaluation of short-term growth. Ann Hum Biol 2001;28:195-206.
9 Bogin B: Patterns of Human Growth, ed 2. Cambridge, Cambridge University Press, 1999.

10 Butler GE, McKie M, Ratcliffe SG: The cyclic nature of prepubertal growth. Ann Hum Biol 1990;17:177-198.

- 11 Hermanussen M, Rol de Lama M, Tresguerres JAF, Grasedyck L, Burmeister J: Short term growth: evidence for chaotic series of mini growth spurts in rat growth. Physiol Behav 1998;64:7-13.

12 Hermanussen M, Seele K: Mini-knemometry: an accurate technique for lower leg length measurements in early childhood. Ann Hum Biol 1997;24:307-313.

-13 Lampl M, Johnson ML, Frongillo EA Jr: Mixed distribution analysis identifies saltation and stasis growth. Ann Hum Biol 2001; 28:403-411.

-14 Hermanussen M, Geiger-Benoit K, Sippell WG: $\mathrm{GH}$ deficient children receiving $\mathrm{GH}$ replacement do not grow during intermittent infectious illness. Acta Paediatr Scand 1986; 75:601-604.

15 Prader A, Tanner JM, Harnack G: Catch-up growth following illness or starvation. An example of developmental canalization in man. J Pediatr 1963;62:646-659.
16 Wit JM, Boersma B: Catch-up growth: definition, mechanisms, and models. J Pediatr Endocrinol Metab 2002;15(suppl 5):12291241.

17 Caino S, Kelmansky D, Adamo P, Lejarraga $\mathrm{H}$ : Short-term growth in healthy infants, schoolchildren and adolescent girls. Ann Hum Biol 2006;33:213-226.

18 Glock M, Hermanussen M, Keller E, Hartmann KK: Gulliver G-100 - A new device to evaluate daily growth measurement in comparison with Harpenden stadiometer. Horm Res 1999;52:287-290.

19 Hermanussen M, Gausche R, Keller A, Kiess W, Brabec M, Keller E: Short-term growth response to $\mathrm{GH}$ treatment and considerations upon the limits of short-term growth predictions. Horm Res 2002;58:71-77.

20 Chu CK, Glad IK, Godtliebsen F, Marron JS: Edge-preserving smoothers for image processing. J Am Stat Assoc 1998;93:526-556.

21 Cole TJ, Green PJ: Smoothing reference centile curves: the LMS method and penalized likelihood. Stat Med 1992;11:1305-1319.

22 Cole TJ: Growth monitoring with the British 1990 growth reference. Arch Dis Child 1997; 76:47-49. 
23 Garza C, de Onis M: Rationale for developing a new international growth reference. Food Nutr Bull 2004;25(1 suppl):S5-S14.

-24 Cole TJ: Body mass index reference curves for the UK 1990. Arch Dis Child 1995;73:2529.

25 Cole TJ: Presenting information on growth distance and conditional velocity in one chart: practical issues of chart design. Stat Med 1998;17:2697-2707.

-26 Waterlow JC, Buzina R, Keller W, Lane JM, Nichaman MZ, Tanner JM: The presentation and use of height and weight data for comparing the nutritional status of groups of children under the age of 10 years. Bull World Health Organ 1977;55:489-498.

-27 Prader A, Largo RH, Molinari L, Issler C: Physical growth of Swiss children from birth to 20 years of age. First Zurich Longitudinal Study of Growth and Development. Helv Paediatr Acta Suppl 1989;52:1-125.

-28 Hermanussen M, Largo RH, Molinari L: Canalisation in human growth: a widely accepted concept reconsidered. Eur J Pediatr 2001;160:163-167.

29 Preece M, Baines MJ: A new family of mathematical models describing the human growth curve. Ann Hum Biol 1978;5:1-24.
30 Molinari L, Hermanussen M: The effect of variability in maturational tempo and midparent height on variability in linear body measurements. Ann Hum Biol 2005;32:679_ 682.

31 Hewitt D, Acheson RM: Some aspects of skeletal development through adolescence. I. Variations in the rate and pattern of skeletal maturation at puberty. Am J Phys Anthropol 1961;19:321-331.

32 Hewitt D, Acheson RM: Some aspects of skeletal development through adolescence. II. The inter-relationship between skeletal maturation and growth at puberty. Am Phys Anthropol 1961;19:333-344.

33 Tanner JM, Whitehouse RH, Marshall WA, Healy MJR, Goldstein H: Assessment of Skeletal Maturity and Prediction of Adult Height (TW2 Method), ed 2. London, Academic Press, 1983.

34 Ramsay JO, Silverman BW: Functional Data Analysis. Berlin, Springer, 1997.

35 Hermanussen M, Meigen C: Phase variation in child and adolescent growth. Int J Biostat 2007;3:1-16.

-36 Aswani N, Taylor CJ, McGaw J, Pickering M, Rigby AS: Pubertal growth and development in cystic fibrosis: a retrospective review. Acta Paediatr 2003;92:1029-1032.
37 Wiedemann B, Steinkamp G, Sens B, Stern M, German Cystic Fibrosis Quality Assurance Group: The German cystic fibrosis quality assurance project: clinical features in children and adults. Eur Respir J 2001;17: 1187-1194.

38 Brundtland GH, Liestøl K, Walløe L: Height, weight and menarcheal age of Oslo schoolchildren during the last 60 years. Ann Hum Biol 1980;7:307-322.

39 Hagen W: Zum Akzelerationsproblem. Dtsch Med Wochenschr 1961;86:220-223.

40 Caino S, Kelmansky D, Adamo P, Lejarraga $\mathrm{H}$ : Short-term growth in head circumference and its relationship with supine length in healthy infants. Ann Hum Biol 2010;37:108116.

41 Thodberg HH, Jenni OG, Caflisch J, Ranke MB, Martin DD: Prediction of adult height based on automated determination of bone age. J Clin Endocrinol Metab 2009;94:48684874

42 Thodberg HH: Clinical review: An automated method for determination of bone age. J Clin Endocrinol Metab 2009;94:2239-2244.

43 Meigen C, Hermanussen M: Automatic analysis of longitudinal growth data on the website willi-will-wachsen.de. Homo 2003;54: 157-161. 